\title{
Observations of Eclipsing Binary EPIC 201826968
}

\author{
UMAR AHMEd BAdAMI ${ }^{1}$, LOUIS GOSART ${ }^{1}$, JAKE NORTH ${ }^{1}$, ANd KALÉE \\ TOCK $^{1}$ \\ ${ }^{1}$ Stanford Online High School, Stanford, CA, USA
}

\begin{abstract}
The eclipsing binary system EPIC 201826968 was imaged using the Las Cumbres Observatory Global Telescope Network with Bessel-B, Bessel-V, SDSS-r' and SDSS-i' filters. AstroImageJ was used to determine the optimal exposure time for the images. We coded a phase-dispersion minimization (PDM) algorithm and compared its result to PyAstronomy's PDM algorithm and both PyAstronomy's and Astropy's Lomb-Scargle algorithms. Our distance PDM algorithm gave a period of 0.3617673 days, while the PyAstronomy PDM gave 0.3617724 days. The Lomb-Scargle algorithms both gave very different periods of near 1.83 days, possibly due to Lomb-Scargle's reliance on a sinusoidal fit. Since Kepler measured a period of 0.3617589 days, and the average period from the Python-coded and PyAstronomy PDM algorithms deviated from it by less than a second, we concluded that the period of eclipsing binary system EPIC 201826968 has not changed since Kepler's observations.
\end{abstract}

()(1) $\odot \bigodot 2020$ Astronomy Theory, Observations and Methods Journal

Keywords: binaries: eclipsing - methods: data analysis - techniques: photometric - telescopes

https://doi.org/10.32374/atom.2020.1.5

\section{INTRODUCTION}

An eclipsing binary is a type of binary star system whose orbital shape and inclination causes one star to block the other from Earth's perspective. These systems can be difficult to observe using traditional optical methods, as many are physically so close together that they appear as one star. Therefore, it is impossible to measure the position angle and separation of the stars in the system. However, their orbits can be analyzed by measuring changes in light intensity that occur during eclipses, when one star passes in front of the other. These data can be used to construct a lightcurve, in which normalized flux is plotted versus phase, where phase is the fraction of orbital period elapsed. Normalized flux is used instead of light intensity in order to scale the graph so that the average flux of data points outside of the eclipse is 1 . This provides a convention by which eclipsing binary systems can be easily compared to each other.

From analyzing the lightcurve, the period of an eclipsing binary system can be determined. A sample lightcurve is shown in Figure 1. Due to their small physical separation, eclipsing binaries generally have periods of less than a few days (Giuricin, Mardirossian, \& Mezzetti, 1983). Repeated measurements of the system's period can help to determine its rate of change, which indicates the extent of mass 
exchange between the stars (Plavec, 1970).

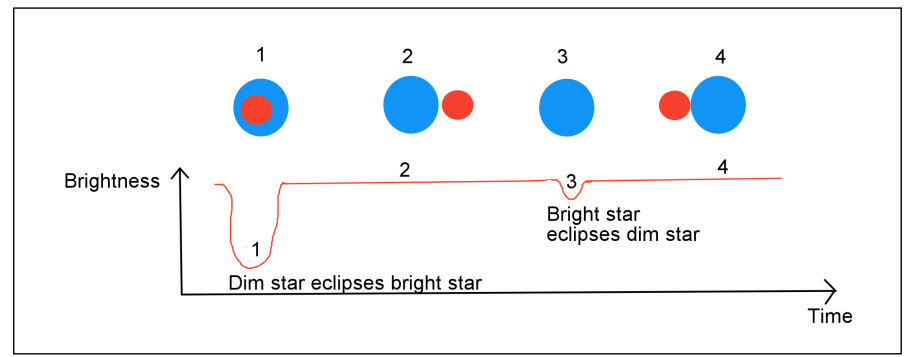

Fig. 1. Sample lightcurve of an eclipsing binary. The blue star is brighter than the red one.

In this paper, the eclipsing binary system EPIC 201826968 is analyzed for a change in period since its last observation. This is done by obtaining timeseries images in four filters (Bessel-B, Bessel-V, SDSS-r ', and SDSS-i'), creating and examining the system's lightcurve, and using period-finding algorithms such as Phase-Dispersion Minimization (PDM) and LombScargle. In the course of this examination, we also investigated the appropriateness of these algorithms for finding the periods of systems such as ours.

\section{ECLIPSING BINARY EPIC 201826968}

Possible star systems to investigate were selected from Kepler K2 Campaign 1 (Kirk et al., 2016). Campaign 1 was chosen because it contains stars that are visible in late January and February, the time during which imaging was conducted. The eclipsing binary EPIC 201826968 was chosen: its properties are shown in Table 1 . Note that two magnitudes are reported because the data in Table 1 are drawn from both the GAIA and Kepler catalogues. The GAIA satellite includes wavelengths between 300 and 1,100 nanometers (Gaia Collaboration et al., 2016), while the Kepler satellite includes a narrower range between 420 and 865 nanometers (Koch et al., 2004).

This system has a secondary eclipse with a depth comparable to that of the primary eclipse, as shown in the lightcurve in Figure 2. The similar depths of the primary and secondary eclipses indicates that the stars may be similar sizes.

\section{TELESCOPE METHODS}

\section{Telescopes}

The Las Cumbres Observatory (LCO) (Brown et al., 2013), which has access to 21 telescopes at 8 sites around the world, was used to image EPIC
Table 1. GAIA and Kepler values for EPIC 201826968 (Kirk et al., 2016; Gaia Collaboration et al., 2016)

$$
\text { RA (degrees) }
$$

Dec (degrees) $+05.85937$

Luminosity (solar luminosities)

Kepler Magnitude $\mathrm{kmag}=11.6430$

Gaia Magnitude

Gmag $=11.605$

Absolute G-band Magnitude

0.9540

Period (days)

0.3617589

Period Error (\%)

0.004

Parallax (mas)

2.9433

Proper Motion (mas/yr)

$-34.732,0.082$

Stellar Effective Temperature (K)

5355

Stellar Radius (solar radii)

1.59

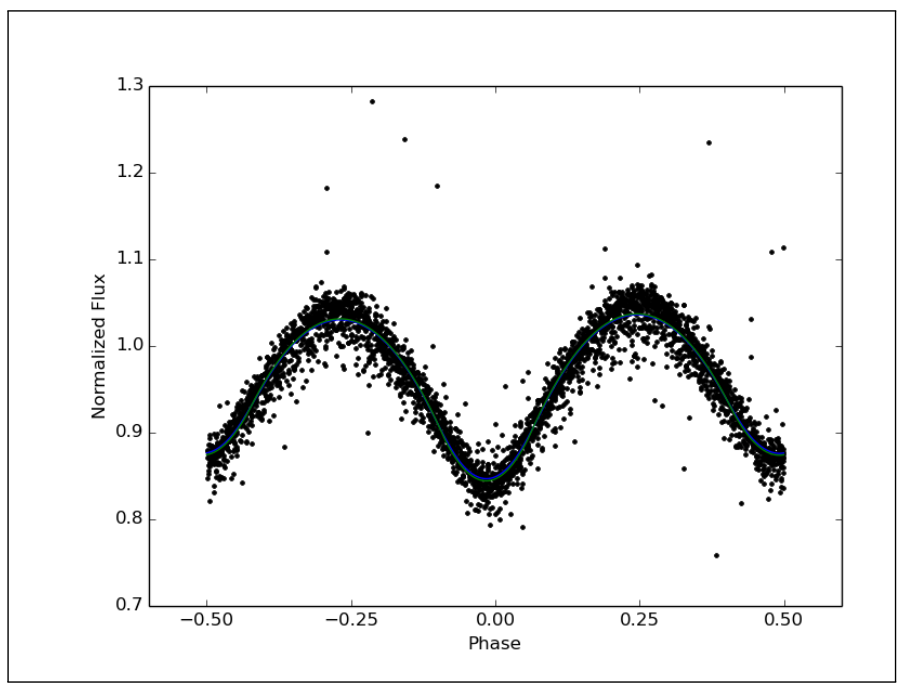

Fig. 2. Existing Kepler lightcurve for EPIC 201826968 (Kirk et al., 2016) 
201826968 . Figure 3 shows one of the 0.4-meter telescopes in the LCO network.

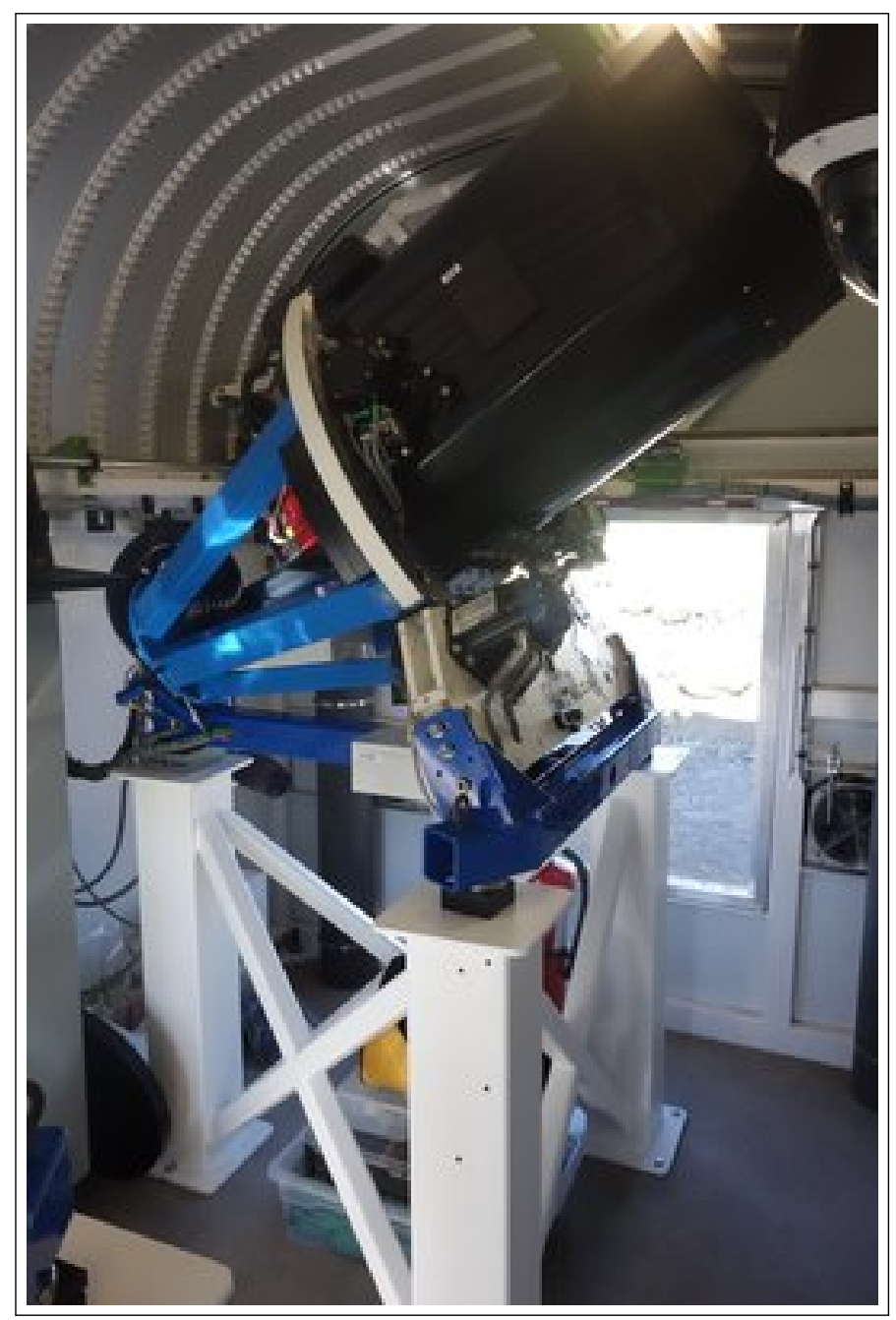

Fig. 3. One of the 0.4-meter telescopes in the LCO network. (Brown et al., 2013)

\section{Exposure Times}

We requested images from the LCOGT network, taken in each of four filters: Bessel-B, Bessel-V, SDSS- $\mathrm{r}^{\prime}$, and SDSS-i'. These images can be found in Supplemental Documents. To find the optimal exposure time for this system on the $0.4 \mathrm{~m}$ LCO telescopes, source-minus-sky analog-digital unit (ADU) counts were evaluated for the star in 8 images from each of the filters, and averaged for each filter. The counts were found using AstroImageJ (AIJ) software (Collins, Kielkopf, Stassun, \& Hessman, 2017). AIJ uses aperture photometry, which sums the ADU counts of each pixel, or fraction of one, within a given circular aperture surrounding the star. A wider background aperture was set around the star to determine the ADU counts of the back- ground, which was then subtracted to determine the integrated source-minus-sky count for the star.

Choosing the exposure time so as to obtain a source-minus-sky count between 100,000 and 200,000 is a reasonable range to ensure that the images have a good signal-to-noise ratio (SNR) without being saturated (Fitzgerald et al., 2018). Initially, we had set an exposure time of 50 seconds for the blue filter, which did not have a high enough source-minussky value. Then, we requested new images with a modified exposure time of 90 seconds for the blue filter: all other exposure times were kept the same. This doubled the source-minus-sky value, which then fit within the desired range. Optimal exposure times and number of returned images per filter are listed in Table 2.

\section{Comparison Stars}

Examining the stars surrounding the target in our images, we decided on 6 comparison stars, or comp stars, which were used to determine the magnitude of the eclipsing system relative to a non-variable source (Roth, 2009). We used comp stars because the target star counts might vary based on exposure time or atmospheric clarity, which would have interfered with our ability to determine the variation due to eclipses if we had used unaltered magnitudes. However, the ratio of target star ADU count to that of a nearby nonvariable star in the same image would be expected to stay constant, because both stars would experience the same variation in their counts from these factors (Buchheim, 2007). This technique is called differential photometry. Additional comp stars can be used to ensure that the chosen comp stars are not themselves variable by comparing them with each other. Out of the six original comp stars, three of them had sourcesky counts under 100,000, which were unsuitable for comparisons due to low SNR, and another had asymmetry that made it look like a double star. Table 3 shows the coordinates of the remaining two comp stars chosen for this study.

Figure 4 shows the difference between the comp stars' magnitudes: since the difference stays relatively constant over the phase of the target system, the quality of the images used and the non-variability of the comp stars can be inferred. The mean differential magnitude was 1.256 , with a standard deviation of 0.019 . 
Table 2. Optimal exposure times for EPIC 201826968 on the $0.4 \mathrm{~m}$ LCO telescopes

\begin{tabular}{ccc}
\hline Filter Color & Exposure Time (seconds) & Number of Returned Images \\
\hline Blue & 90 & 116 \\
Red & 20 & 116 \\
Infrared & 30 & 97 \\
Visible & 30 & 111 \\
\hline
\end{tabular}

Table 3. Comp star coordinates

\begin{tabular}{ccc}
\hline Comp Star Number & RA (Degrees) & Dec (Degrees) \\
\hline 1 & 178.49805 & 5.783633 \\
2 & 178.41852 & 5.828768 \\
\hline
\end{tabular}

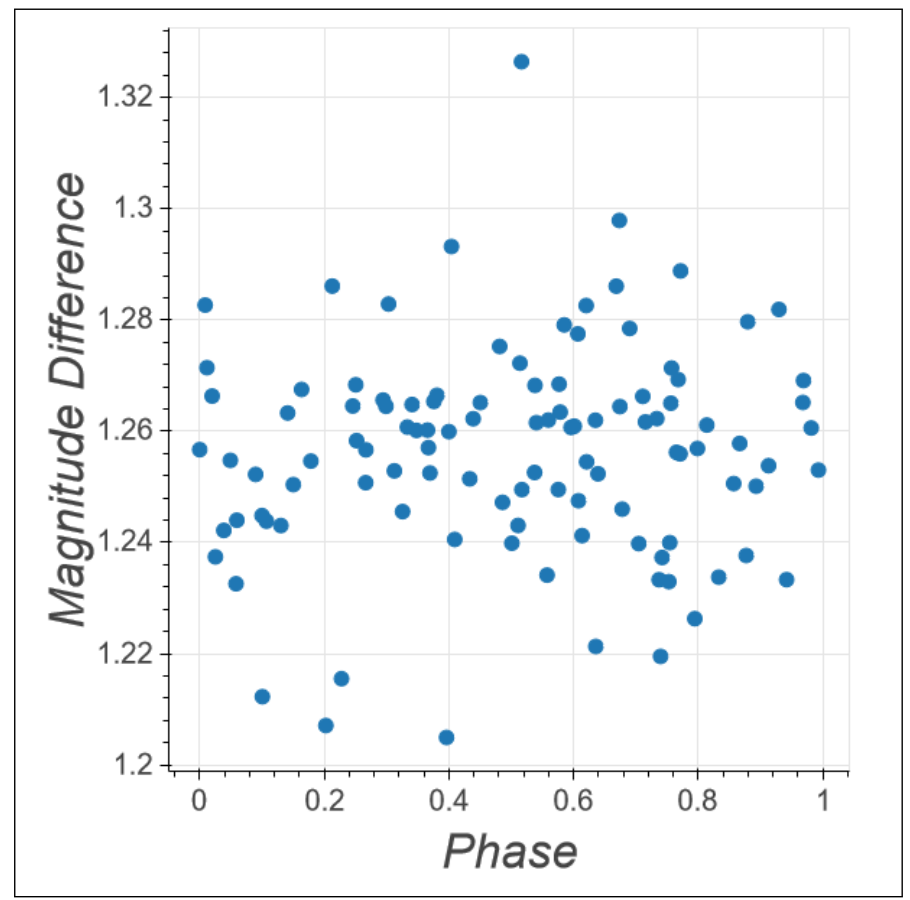

Fig. 4. Plot of the difference between comp star magnitudes over phase of the target system, determined using the Kepler period.

\section{PERIOD DETERMINATION}

\section{Data Transformations}

We took a series of images in various filters from LCO (see Supplemental Documents for the image files used). These images were processed with various photometries by the Our Solar Siblings (OSS) pipeline (Fitzgerald, 2018). We noted their modified Julian dates (MJDs) and the ADU counts of the target and comparison stars. To turn the MJDs of the observations into phase, the difference between the observation date and the initial observation date was divided by the period, and the portion after the decimal point in the quotient was retained. In other words:

$$
P(D)=\frac{D-D_{0}}{P} \bmod 1,
$$

where $P(D)$ is the phase, $D$ is the observation date, $D_{0}$ is the date of the first observation, $P$ is the assumed period, and mod is the remainder operator.

The differential magnitude of each observation is calculated by multiplying -2.5 by the base- 10 logarithm of the count of the target star divided by the count of the comparison star. This can be represented as:

$$
F=-2.5 \log _{10} \frac{S_{t}}{S_{c}}
$$

where $F$ is the differential magnitude, $S_{t}$ is the source-minus-sky of the target star for the observation, and $S_{c}$ is the source-minus-sky of the comparison star in the same observation.

Phase versus differential magnitude plots were made for all images with all filter color and photometry type combinations. Based on the plots in Supplemental Documents, we determined that data taken with Bessel-B filter and sex (source extractor) photometry gave us the visually clearest lightcurves. This technique is similar to that employed by Altunin and Caputo (Altunin \& Caputo, 2019). Bessel-B filter 
and sex photometry were hence used in all following analysis (Bertin \& Arnouts, 1996).

\section{Distance PDM}

We coded a Python version of the PDM Minimum Distance method to estimate the period of our system based on (Dworetsky, 1983). Using an assumed period, we obtained the phase of our system based on a phase of 0 at the start date of our observations. Using our differential magnitude, calculated phase, and the distance formula for points on a 2-dimensional graph, we calculated the distance between consecutive points in flux-phase space, and added them together to get one total sum. The smaller our sum, the closer the data points are, the more accurate our lightcurve, and the closer our estimate of the period is to its actual value. For our data, the Minimum Distance algorithm gave us a closest period of $0.3618 \pm 0.0004$ days, larger than the Kepler period by 0.73 seconds. The error was computed using the width of the primary peak at half maximum.

\section{PyAstronomy PDM}

We used the PyAstronomy version of Wolk's Phase Dispersion Minimization (PDM) Standard Deviation algorithm as a method of estimating the period of our eclipsing binary system (Wolk, 1996; Scanner and PDM Class, accessed 2018). In this method, the phase of each observation is determined by the guessed period and the starting date of the observations. The data is then divided into 10 sections by phase (the phase bins are 0.0-0.1, 0.1-0.2,...0.9-1.0). The standard deviations of the fluxes within each bin are summed, and then divided by the standard deviation of the entire data set, yielding a quantity known as theta. The smaller the theta value, the closer the data points are to each other, and the closer its estimate of the period is to the true period of the system. For our data, the PDM Standard Deviation algorithm gave a closest period of $0.3618 \pm 0.0004$ days, larger than the Kepler period by 1.17 seconds.

\section{Astropy and PyAstronomy Lomb-Scargle methods}

We also used the Astropy and PyAstronomy versions of the Lomb-Scargle periodogram, originally developed independently by Lomb and Scargle (Lomb, 1976; Scargle, 1982). The Lomb-Scargle method attempts to perform a sinusoidal fit on a large set of observational data, taken over a range of times. The algorithm determines the probability, or power, of a certain period by measuring the quality of the sinusoidal fit. The period with the largest power is thus the most likely, according to Lomb-Scargle. For our data, the Astropy version of Lomb-Scargle reported a most likely period of $1.838 \pm 0.044$ days, greater than Kepler's by over 1.47 days. Similarly, the PyAstronomy version gave a period of $1.832 \pm 0.013$ days, which showed a similar discrepancy from Kepler's measurement.

\section{Comparison of Algorithms: Periods and Statistics}

As shown in Table 4, the period found by Kepler is very similar to the periods found by the Distance PDM and PyAstronomy PDM methods. However, these periods are very different from those found by both Lomb-Scargle algorithms. As explained above, for PDM-analyzed periods lower values indicate a more likely period, while for Lomb-Scargle-analyzed periods higher values indicate a more likely period as determined by the respective algorithm.

Likewise, as shown in Figure 5, the lightcurve made with Kepler's period is very similar to those with the Distance PDM and PyAstronomy PDM periods. However, both Lomb-Scargle algorithms give extremely different and noisy lightcurves.

Figure 6 shows the power graph of each periodcalculation method, where "power" refers to the likelihood of a given period. Both PDM methods have very clearly-defined peaks at their predicted periods and the remainder of their graphs descend to lowlevel noise. The power graphs of both Lomb-Scargle methods also demonstrate peaks near their predicted periods, but the peaks are quite noisy and, in the case of the AstroPy Lomb-Scargle algorithm, other similarly-large peaks also occur closer to the Kepler and PDM-derived periods.

\section{DISCUSSION OF RESULTS}

Figures 7 and 8 and show a small selection of lightcurves from Supplemental Documents, which used images taken with various filters and photometry types to produce lightcurves of EPIC 201826968 using the Kepler period. They demonstrate that the "visually cleanest" lightcurve arises from the B filter and sex photometry types, which was the conclusion drawn in Section .

As shown in Table 4 and Figure 5, the LombScargle-derived periods and lightcurves are extremely different from Kepler's, while the PDM algorithm periods are very similar to Kepler's. Likewise, Figure 6 


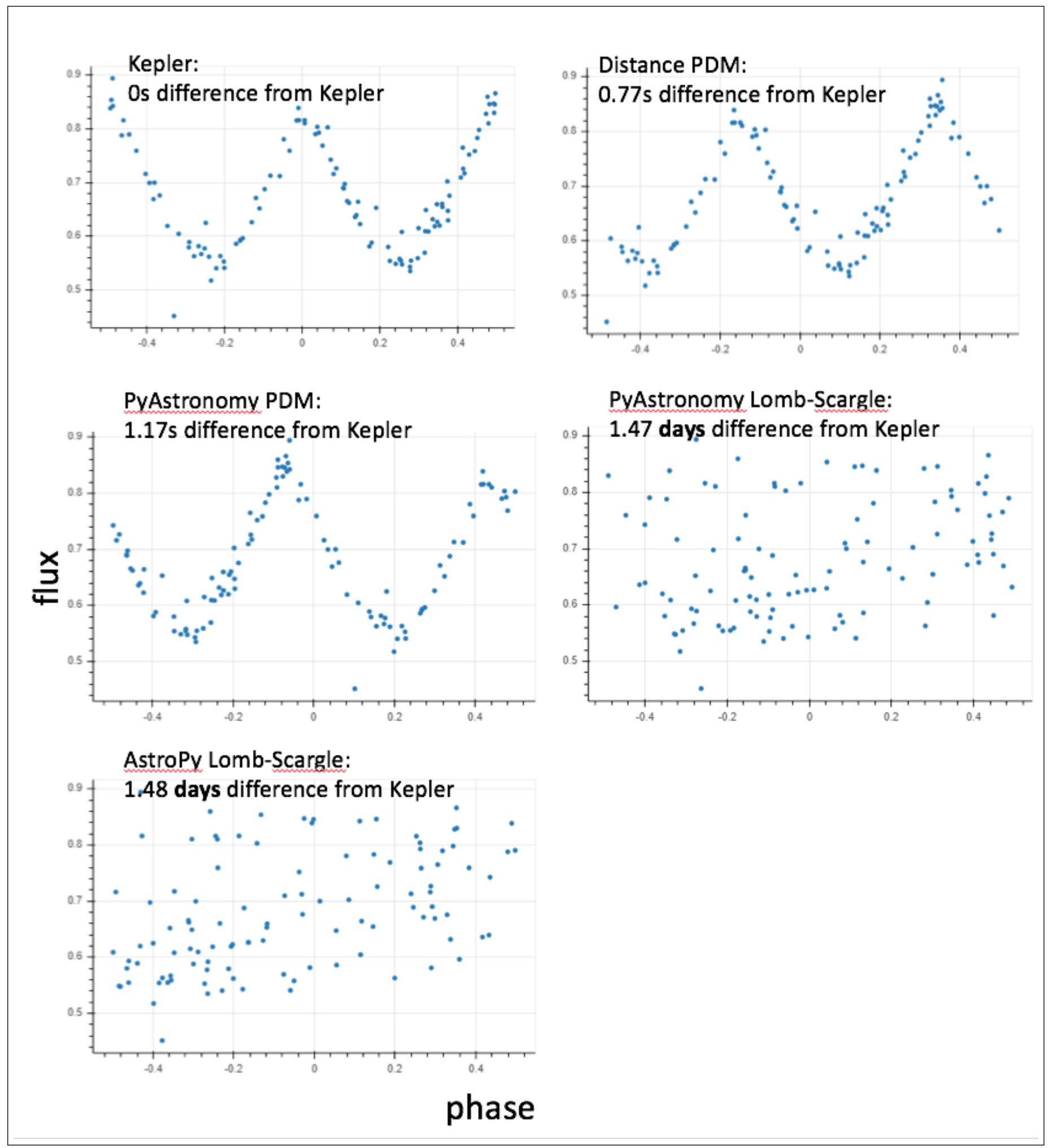

Fig. 5. Each period with its period difference from Kepler and a lightcurve. 
Table 4. Each period method with its period and the measured period's statistics from other methods.

\begin{tabular}{|c|c|c|c|c|c|}
\hline Period Method & Period (days) & $\begin{array}{l}\text { Total } \\
\text { Distance of } \\
\text { this Period } \\
\text { Calculated } \\
\text { with our } \\
\text { Images }\end{array}$ & $\begin{array}{c}\text { PyAstronomy } \\
\text { PDM Theta } \\
\text { of this } \\
\text { Period } \\
\text { Calculated with our } \\
\text { Images (10 bins) }\end{array}$ & $\begin{array}{c}\text { Astropy } \\
\text { Lomb-Scargle } \\
\text { Power of this } \\
\text { Period } \\
\text { Calculated with } \\
\text { our Images }\end{array}$ & $\begin{array}{c}\text { PyAstronomy } \\
\text { Lomb-Scargle } \\
\text { Power of this } \\
\text { Period } \\
\text { Calculated } \\
\text { with our Images }\end{array}$ \\
\hline Kepler & 0.3617589 & 3.54 & 0.190 & 0.0267 & 0.00561 \\
\hline Distance PDM & 0.3617673 & 3.33 & 0.143 & 0.0273 & 0.00561 \\
\hline PyAstronomy PDM & 0.3617724 & 3.47 & 0.143 & 0.0275 & 0.00561 \\
\hline $\begin{array}{c}\text { Astropy } \\
\text { Lomb-Scargle }\end{array}$ & 1.8376291 & 10.1 & 0.855 & 0.125 & 0.126 \\
\hline $\begin{array}{l}\text { PyAstronomy } \\
\text { Lomb-Scargle }\end{array}$ & 1.8321272 & 11.9 & 0.905 & 0.126 & 0.125 \\
\hline
\end{tabular}

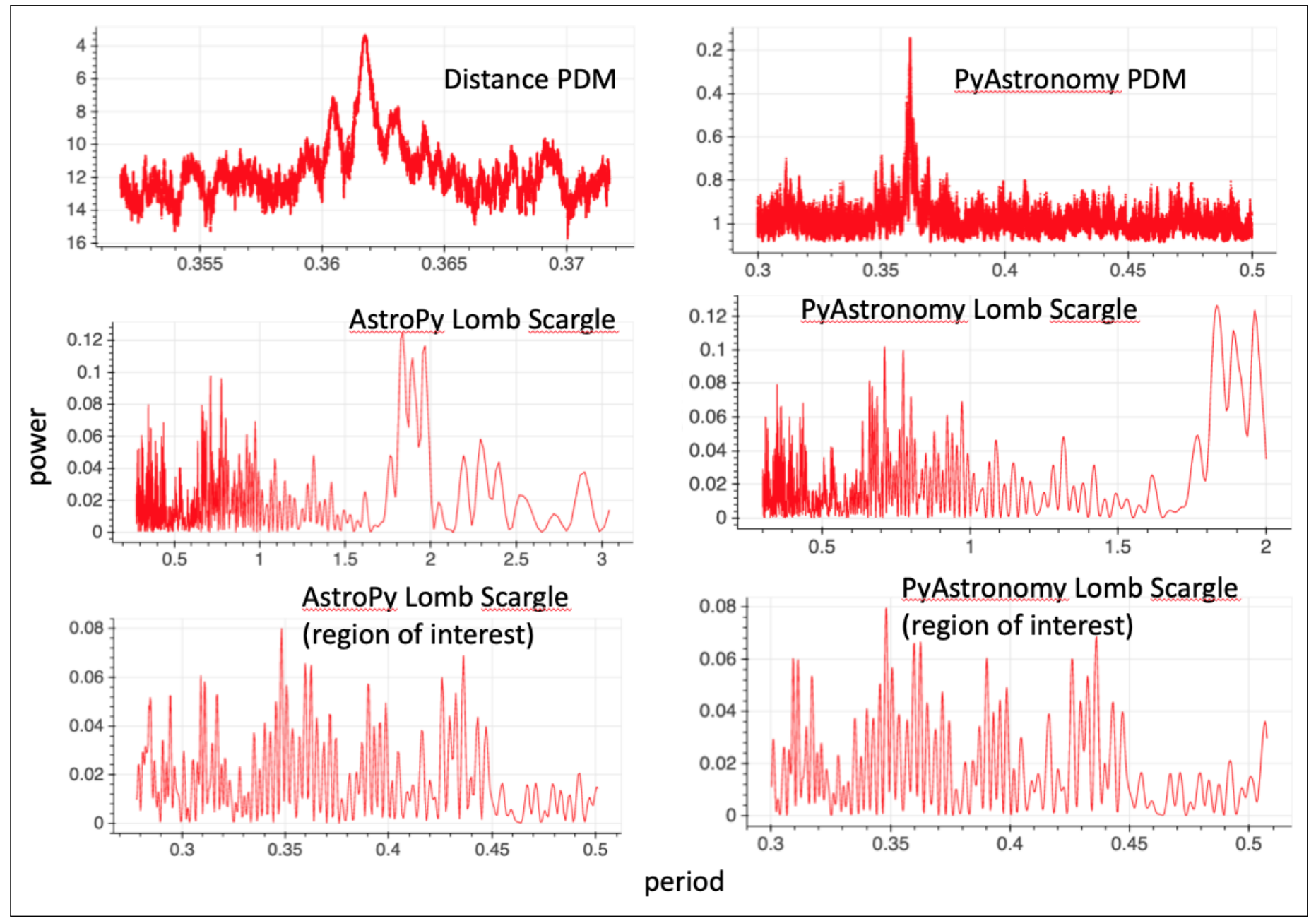

Fig. 6. Each period-calculation method with its power graph. As lower statistics for the PDM algorithms imply a greater likelihood of a correct period, the y-axes for those graphs go from higher to lower values. 


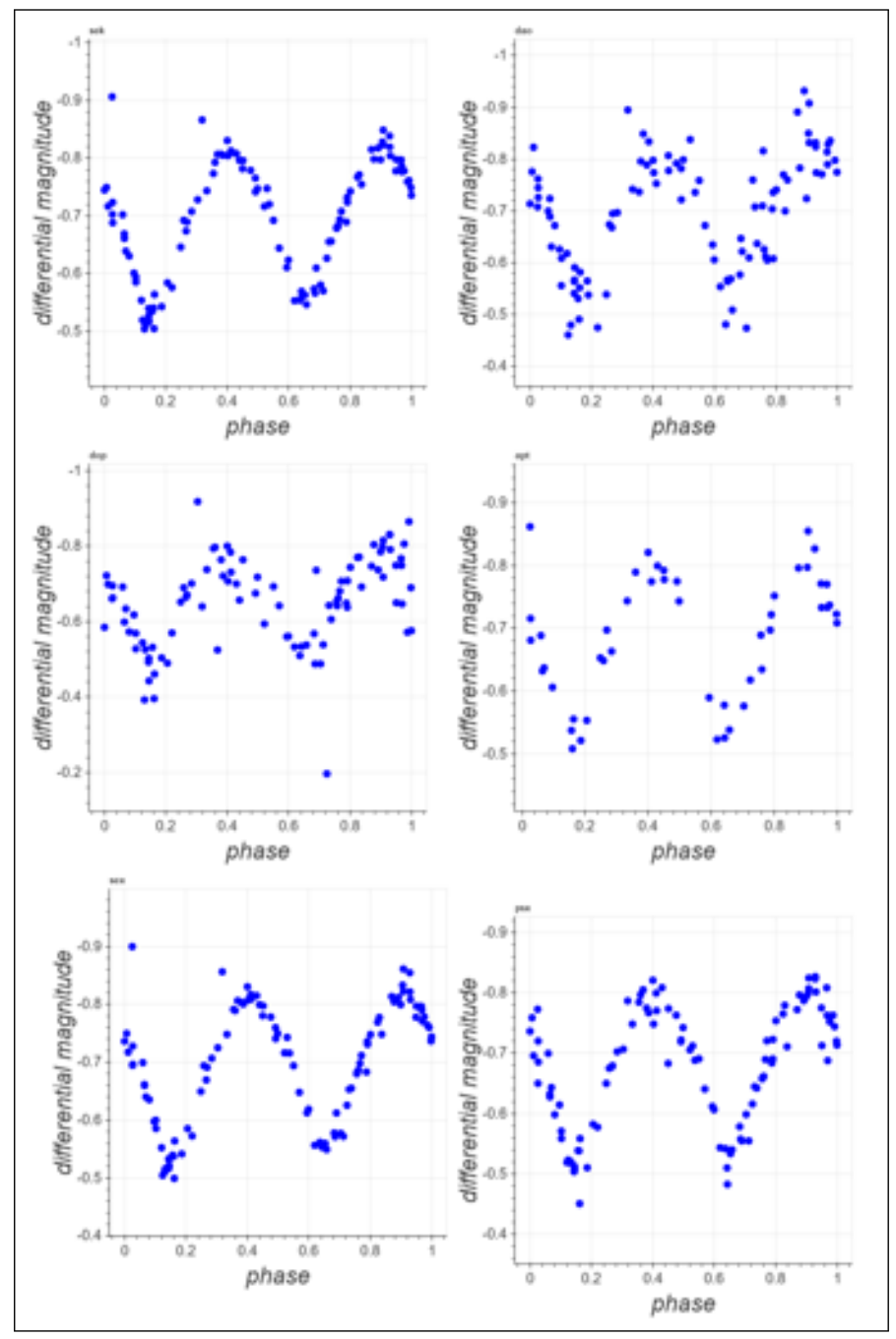

Fig. 7. Lightcurves using B-filter images in all six photometry types.

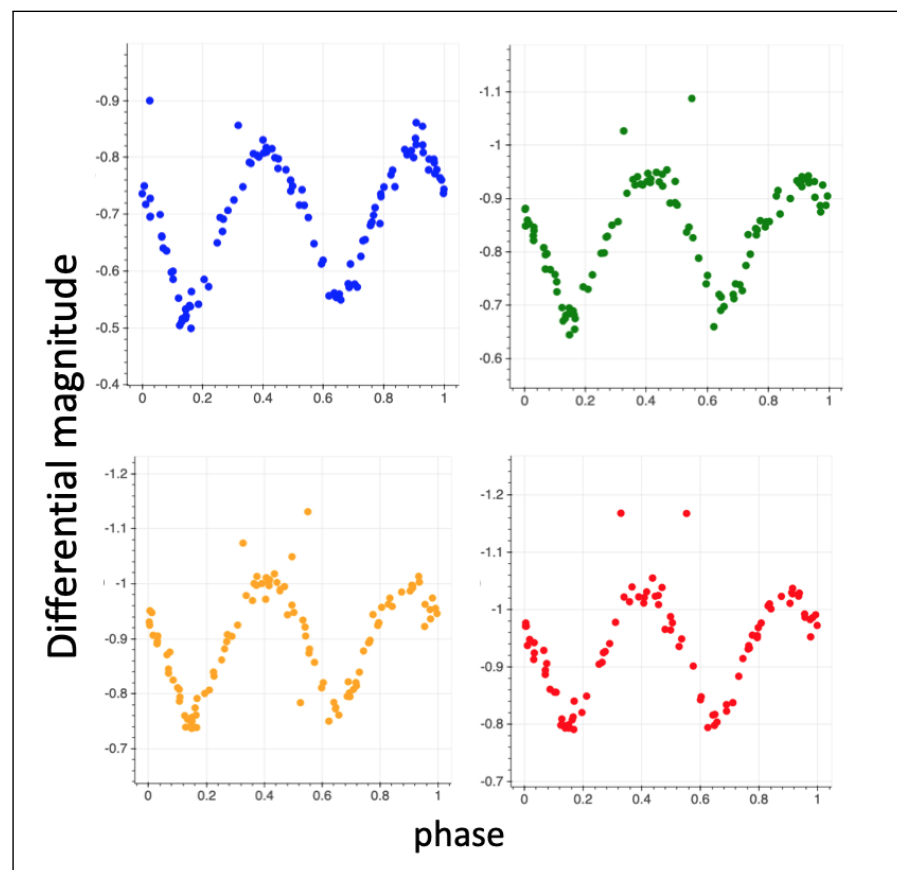

Fig. 8. Lightcurves using images in all four filter types processed with sex photometry.

demonstrates much cleaner power distributions for the PDM algorithms than for the Lomb-Scargle ones. Thus, Lomb-Scargle methods appear to be noisier and more inaccurate compared to the PDM methods when applied to measurements of our system. In Table 5, the period of each algorithm is shown together with its error. Except for the PyAstronomy LombScargle Algorithm, whose error was given directly by the PyAstronomy Lomb-Scargle module, the error is estimated as the full width at half maximum of the corresponding peak in the power plot. As is evident from these values, both our Distance PDM period and the PyAstronomy PDM period are not statistically different from the Kepler algorithm's period, while both Lomb-Scargle algorithms give statistically different results.

According to Lomb, the Lomb-Scargle algorithm works by "fitting sine waves by least-squares to the data" (Lomb, 1976). This statement is also reflected in the Astropy and PyAstronomy descriptions of their slightly modified Lomb-Scargle algorithms. (Mayangsari, Priyatikanto, \& Putra, 2014) have determined that PDM algorithms are more accurate for systems with non-sinusoidal fits, which may be the case with EPIC 201826968. Therefore, the LombScargle algorithm's discrepancy may be explained by the non-sinusoidal nature of this system. 
Table 5. Each period method with its period and the measured period's errors.

\begin{tabular}{ccc}
\hline Period Method & Period (days) & $\begin{array}{c}\text { Period Error } \\
\text { (days) }\end{array}$ \\
\hline Kepler & 0.3617589 & 0.000016 \\
Distance PDM & 0.3617 & 0.0004 \\
PyAstronomy PDM & 0.3618 & 0.0004 \\
$\begin{array}{c}\text { Astropy } \\
\text { Lomb-Scargle } \\
\text { PyAstronomy }\end{array}$ & 1.838 & 0.044 \\
Lomb-Scargle & 1.832 & 0.013 \\
\hline
\end{tabular}

\section{CONCLUSION}

Using the distance and PyAstronomy PDM methods to determine the period of EPIC 201826968, we conclude that the current period of EPIC 201826968 is $0.36177 \pm 0.00056$ days (calculated by averaging the Distance PDM and PyAstronomy PDM periods and adding their errors in quadrature). Our period deviates from the previously recorded Kepler period by about 1 second. The Lomb-Scargle algorithm is inappropriate for determining the period of our system, possibly due to our system's non-sinusoidal lightcurve. Further observations of this system would be significantly beneficial to the scientific community to determine whether the period is changing. As of this writing, the period of EPIC 201826968 has changed by less than 48.3 seconds since the time of the observations made by the Kepler space telescope.

\section{ACKNOWLEDGMENTS}

The advice, assistance and information shared with us by Michael Fitzgerald of Our Solar Siblings was useful to this work. This research made use of Astropy, a community-developed core Python package for Astronomy (Astropy Collaboration et al., 2013, 2018). This work also made use of PyAstronomy. This work makes use of observations from the LCOGT network (Brown et al., 2013). This research has made use of the VizieR catalogue access tool, CDS, Strasbourg, France. The original description of the VizieR service was published in A\&AS 143, 23. This work has made use of data from the European Space Agency (ESA) mission Gaia, processed by the Gaia Data Processing and Analysis Consortium (DPAC). Funding for the DPAC has been provided by national institutions, in particular the institutions participating in the Gaia
Multilateral Agreement. We would like to thank the Las Cumbres Observatory for allowing researchers to use their many telescopes, and Karen Collins for making the AstroImageJ software freely available for researchers to use. We would also like to thank the Our Solar Siblings pipeline for processing and performing photometry on all of our images.

\section{SUPPLEMENTAL DOCUMENTS}

All supplemental documents are presented below.

See this for the images used in this project.

See this for the code used to plot the difference over phase of comp star magnitudes.

See this for lightcurves plotted with the Kepler period with all photometries and all filter types.

See this for supporting content for the lightcurveplotting Python code.

See this for supporting content for the Distance PDM algorithm Python code.

See this for supporting content for the PyAstronomy PDM algorithm Python code.

See this for supporting content for the Astropy LombScargle algorithm Python code.

See this for supporting content for the PyAstronomy Lomb-Scargle algorithm Python code.

\section{REFERENCES}

Altunin, I., \& Caputo, R. (2019). Period of Eclipsing Binary EPIC 201458798. Astronomy Theory, Observations and Methods, submitted.

Astropy Collaboration, Price-Whelan, A. M., Sipócz, B. M., Günther, H. M., Lim, P. L., Crawford, S. M., ... Astropy Contributors (2018, September). The Astropy Project: Building an Openscience Project and Status of the v2.0 Core Package. , 156, 123.

Astropy Collaboration, Robitaille, T. P., Tollerud, E. J., Greenfield, P., Droettboom, M., Bray, E., ... Streicher, O. (2013, October). Astropy: A community Python package for astronomy. , 558, A33. 
Bertin, E., \& Arnouts, S. (1996, June). SExtractor: Software for source extraction. Astronomy and Astrophysics Supplement Series, 117, 393-404.

Brown, T. M., Baliber, N., Bianco, F. B., Bowman, M., Burleson, B., Conway, P., ... Willis, M. (2013, Sep). Las Cumbres Observatory Global Telescope Network. , 125(931), 1031. doi:

Buchheim, R. K. (2007). The sky is your laboratory: Advanced astronomy projects for amateurs.

Collins, K. A., Kielkopf, J. F., Stassun, K. G., \& Hessman, F. V. (2017, January). Astroimagej: Image processing and photometric extraction for ultra-precise astronomical light curves. The Astronomical Journal, 153. Retrieved from http://iopscience.iop.org/ article/10.3847/1538-3881/153/2/77

Dworetsky, M. M. (1983, June). A period-finding method for sparse randomly spaced observations or "How long is a piece of string ?". , 203, 917-924.

Fitzgerald, M. T. (2018). The our solar siblings pipeline: Tackling the data issues of the scaling problem for robotic telescope based astronomy education projects. RTSRE, 1(1), 347-358.

Fitzgerald, M. T., McKinnon, D. H., Danaia, L., Cutts, R., Salimpour, S., \& Sacchi, M. (2018). Our solar siblings. a high school focussed robotic telescope-based astronomy education project. RTSRE, 1(1), 221-235.

Gaia Collaboration, Prusti, T., de Bruijne, J. H. J., Brown, A. G. A., Vallenari, A., Babusiaux, C., ... Zschocke, S. (2016, Nov). The Gaia mission. , 595, A1. doi:

Giuricin, G., Mardirossian, F., \& Mezzetti, M. (1983, March). The period distribution of eclipsing binary systems. , 119, 218-226.

Kirk, B., Conroy, K., Prša, A., Abdul- Masih, M., Kochoska, A., Matijevič, G., ... Borucki, W. (2016, March). Kepler Eclipsing Binary Stars. VII. The Catalog of Eclipsing Binaries Found in the Entire Kepler Data Set. , 151, 68. doi:

Koch, D. G., Borucki, W., Dunham, E., Geary, J., Gilliland, R., Jenkins, J., ... Weiss, M. (2004, Oct). Overview and status of the Kepler Mission. In J. C. Mather (Ed.), (Vol. 5487, p. 14911500). doi:

Lomb, N. R. (1976, February). Least-Squares Frequency Analysis of Unequally Spaced Data. , 39, 447-462.

Mayangsari, L., Priyatikanto, R., \& Putra, M. (2014).
On the period determination of asas eclipsing binaries. AIP Conference Proceedings, 1589(1), 37-41.

Plavec, M. (1970). Mass exchange in binary stars. Publications of the Astronomical Society of the Pacific, 82(489), 957-995. Retrieved from http://www.jstor.org/stable/40676541

Roth, G. D. (2009). Handbook of practical astronomy. Scanner and PDM Class. (accessed 2018). Retrieved from https://www.hs.uni-hamburg.de/DE/ Ins/Per/Czesla/PyA/PyA/pyTimingDoc/ pyPDMDoc/classes.html

Scargle, J. D. (1982, December). Studies in astronomical time series analysis. II. Statistical aspects of spectral analysis of unevenly spaced data. , 263, 835-853.

Wolk, S. J. (1996). Phase Dispersion Minimization. Retrieved from http://hea-www.harvard . edu/ swolk/thesis/period/node2.html 